\title{
MHD Simulation Of Laboratory Jets
}

\section{O.D. Toropina*}

Space Research Institute, 84/32 Profsoyuznaya Str, Moscow, 117997, Russia

E-mail: toropinaliki.rssi.ru

\section{G.S. Bisnovatyi-Kogan}

Space Research Institute, 84/32 Profsoyuznaya Str, Moscow, 117997, Russia

\section{S.G. Moiseenko}

Space Research Institute, 84/32 Profsoyuznaya Str, Moscow, 117997, Russia

We present the results of numerical simulations of the formation and development of magnetized jets. We simulate a plasma flow, for which we chose a numerical method, boundary and initial conditions. We investigate the picture of the flow for different magnetic field configurations. We compared these results with the experiment on a NEODIM laser installation. In the absence of a magnetic field, a substantial expansion of the jet occurs. In the presence of a strong poloidal magnetic field, the jet expands only slightly, which confirms the possibility of a magnetic collimation mechanism for astrophysical jets. Under certain values of the magnetic field a ring structure may form. The opening angle of these simulated structures approximates the results of the laboratory experiment.

High Energy Phenomena in Relativistic Outflows VII - HEPRO VII

9-12 July 2019

Facultat de Física, Universitat de Barcelona, Spain

\footnotetext{
* Speaker.
} 


\section{Introduction}

The processes of formation of astrophysical jets are one of the most interesting problems of astrophysics [1]. The study of astrophysical jets is conducted in several directions: observations in the optical, X-ray and radio bands and multidimensional numerical MHD simulation. The simulation must take into account the entire complex nature of relativistic jets, including the gravitational field and the magnetic field of the central object. Recently, the methods of investigating relativistic jets have been supplemented with methods of laboratory astrophysics. They allow the creation of plasma jets with the help of lasers and make it possible to investigate in the laboratory conditions the structure, evolution, and features of the structure of jets.

The equations of magnetic hydrodynamics describing the formation and evolution of jets are nonlinear even in the one-dimensional case. For the theoretical description of the processes occurring in jets, it is necessary to apply methods of multidimensional numerical simulation. Multidimensional numerical calculations make it possible to obtain a detailed picture of the flow of matter in the jet and the shock-wave structure of the flow. Numerical simulation allows calculations to be performed both for initial data corresponding to astrophysical jets, and for a set of parameters from a laboratory experiment.

Laboratory research is conducted by several groups of scientists. Fua, Liang, Tzeferacos and Lamb work on the creation of a magnetized supersonic laboratory jet. In parallel they perform numerical simulation of the experiment using the two-dimensional FLASH code [2]. Experiments on laboratory modeling of astrophysical jets were carried out at the Kurchatov Institute on the installation of the plasma focus PF-3 by Krauz at al. [3]. Pikuz at al. [4] presented the results of a laboratory laser experiment and the numerical simulation of a magnetized jet ejected from a young stellar object. The collimated jet in these calculations is obtained due to the presence of a poloidal magnetic field.

\section{Experiment}

The purpose of the study is numerical modeling and analysis of the results of a laboratory experiment simulating the formation of cosmic relativistic plasma jets on the $10 \mathrm{TW}$ picosecond NEODIM laser installation (TsNIIMASH, Korolev, Russia) [5].

The laser unit has the following parameters of the laser pulse: energy up to $10 \mathrm{~J}$, wavelength $1.055 \mathrm{mkm}$, duration $1.5 \mathrm{ps}$, laser radiation contrast about $10^{7}$. The focusing system based on an off-axis parabolic mirror with a focal length of $20 \mathrm{~cm}$ provides a concentration of at least $40 \%$ of the energy of the laser beam into a $15 \mu \mathrm{m}$ spot and a peak intensity of $2 \times 10^{18} \mathrm{~W} / \mathrm{cm}^{2}$.

The laboratory experiment consists of the following: a cylindrical chamber is placed a foil of $\mathrm{Cu}$ or $\mathrm{Ta}$ with a thickness of $30 \mu \mathrm{m}$ and $50 \mu \mathrm{m}$ as a target. On the opposite side are track detectors CR-39. The energy $10 \mathrm{~J}=6.24 \times 10^{19} \mathrm{eV}$ is uniformly and instantaneously applied to the central region of the target with a diameter of $10 \mu \mathrm{m}$. As a result of heating the target, two directional plasma ejections appear in the form of symmetrical jets. In our numerical simulation, only one of them is considered.

In the experiment, clearly distinct ring structures formed on track detectors by protons with energies in the range of $0.8 \div 1.7 \mathrm{MeV}$ are observed. An example is shown in Figure 1 . 

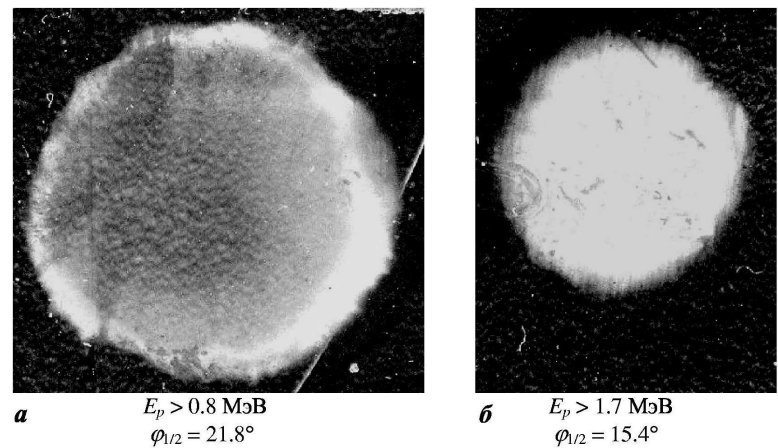

Figure 1: A section of the jet as it impacts the detector: images of proton beams with (a) energies $E_{p}>$ $0.8 \mathrm{MeV}$ and $\phi_{1 / 2}=21.8^{\circ}$ and with (b) $E_{p}>1.7 \mathrm{MeV}$ and $\phi_{1 / 2}=15.4^{\circ}$ at the CR-39 track detectors for a $\mathrm{Cu}$ target with thickness $50 \mu \mathrm{m}$.

\section{Similarity criteria}

The scale of physical processes occurring in astrophysical jets is very different from those in jets that occur when a laser pulse is applied to a target. However, the application of similarity criteria makes it possible to obtain a correspondence between a number of characteristics of an astrophysical and a laboratory jet. The use of such similarity criteria is convenient both for direct comparison of laboratory and observational data, and for multidimensional numerical simulation. Investigation of the similarity criteria for comparing the results of laboratory experiments and astrophysical processes has been devoted to many works, see, for example, [6]. Despite the significant difference in the physical parameters of laboratory and astrophysical jets, there are scaling criteria that allow to connect these quantities:

\begin{tabular}{|l|l|l|}
\hline $\begin{array}{l}\text { Laboratory jet } \\
\text { on NEODIM } \\
\text { installation [5] }\end{array}$ & $\begin{array}{l}\text { Laboratory } \\
\text { jet after } \\
\text { scaling }\end{array}$ & $\begin{array}{l}\text { Jets from } \\
\text { young stars }\end{array}$ \\
\hline$x=0.1-1 \mathrm{~cm}$, & $(3 \div 30) \times 10^{17} \mathrm{~cm}$, & $\sim 3 \times 10^{17} \mathrm{~cm}$, \\
$t=10^{-8} \div 10^{-7} s$, & $3 \times\left(10^{10} \div 10^{9}\right) s$, & $\sim 3 \times 10^{10} \mathrm{~s}$, \\
$v=10^{7} \mathrm{~cm} / \mathrm{s}$, & $10^{7} \mathrm{~cm} / \mathrm{s}$, & $(2 \div 5) \times 10^{7} \mathrm{~cm} / \mathrm{s}$, \\
$\rho=10^{-4} \mathrm{~g} / \mathrm{cm}^{3}$, & $10^{-23} \mathrm{~g} / \mathrm{cm}^{3}$, & $10^{-23} \mathrm{~g} / \mathrm{cm}^{3}$, \\
$n=2 \cdot 10^{19} \mathrm{~cm}^{-3}$, & $2 \mathrm{~cm}^{-3}$, & $(1 \div 100) \mathrm{cm}^{-3}$, \\
$T=10^{4} \mathrm{~K}$, & $10^{4} \mathrm{~K}$, & $(1 \div 6) \times 10^{4} \mathrm{~K}$ \\
$H=10^{8} \div 10^{9} \mathrm{Gs}$, & $1 \div 10 \mathrm{Gs}$ & $1 \div 100 \mathrm{Gs}$ \\
\hline
\end{tabular}

As a result of scaling, we get a satisfactory similarity of a number of jets parameters expiring from the young stars and the jet obtained on the NEODIM laser installation. In more detailed this method is described in our recent paper [7]. 


\section{Numerical model}

During the simulation, the processes of laser heating, melting, and evaporation of the target material were not considered in detail. A considerable amount of work has been devoted to the study of laser ablation processes. For example, Mazhukin et al. investigated nanosecond laser ablation in subcritical and transcritical modes [8]. They also conducted a simulation of laser explosive effervescence of metal [9]. We assume that the ablation process has already happened, the substance has evaporated from the surface of the target. We take these processes into account when determining the initial and boundary conditions.

For numerical simulation of the experiment, the system of equations of magnetohydrodynamics [10] was used. In the future, to more accurately describe the processes occurring with the target material as a result of high-power laser irradiation, it seems necessary to combine MHD and the kinetic approach. But at the initial stage of modeling, we can confine ourselves to the system of MHD equations.

In the simulation an external constant poloidal magnetic field directed along the normal to the plate. Simulations were carried out for different values of the magnetic field. A single-component plasma flow was studied. We investigated the region and shape of the flow at various distances from the target and at different times, the spatial distribution of plasma density and energy and the magnetic field. A scheme of the simulation region can be found in our paper [7].

\subsection{Simulation region}

The analysis of the experiment showed that the formation of the jet is associated with the heating of the plate, and the geometry of the incident laser beam plays no part. Because of this, the mathematical model of jet formation in the experiment can be constructed in axisymmetric approximation, assuming that the spot heated by the laser has the form of a circle. To simulate the problem, an inertial cylindrical coordinate system $(r, \phi, z)$ is used. The center of the coordinate system is placed in the middle of the target. The orientation of the coordinate system was chosen so that the $z$ axis is parallel to the vector of the poloidal magnetic field $H$ and perpendicular to the target. The problem assumes axial symmetry in the distribution of all the macroscopic quantities $\rho, T, v, H, \partial / \partial \phi=0$, but all three components of the velocity $v$ and magnetic fields $H$.

Simulation is carried out in half of the coordinate plane $r-z$. The problem uses a uniform cell size grid, the coordinates of the grid nodes lie in the range $0 \leq r \leq R_{\max }, 0 \leq z \leq Z_{\max }$. Simulation is performed on a uniform grid $(r, z)$ with the number of cells $N_{R} \times N_{Z}=513 \times 1026$.

The target (the central region of the plate to which the energy is applied) is a homogeneous cylinder of radius $R_{d}$, of thickness $Z_{d}$. In this case, $R_{d}<<R_{\max }, Z_{\max }$ and $Z_{d}<<R_{\max }, Z_{\max }$.

\subsection{The system of equations}

We use an axisymmetric, resistive MHD code. The code incorporates the methods of local iterations and flux-corrected-transport developed by Zhukov, Zabrodin, Feodoritova [11]. The flow is described by the resistive MHD equations in the absence of gravity:

$$
\frac{\partial \rho}{\partial t}+\nabla \cdot(\rho \mathbf{v})=0
$$




$$
\begin{gathered}
\rho \frac{\partial \mathbf{v}}{\partial t}+\rho(\mathbf{v} \cdot \nabla) \mathbf{v}=-\nabla p+\frac{1}{c} \mathbf{J} \times \mathbf{H} \\
\frac{\partial \mathbf{H}}{\partial t}=\nabla \times(\mathbf{v} \times \mathbf{H})+\frac{c^{2}}{4 \pi \sigma} \nabla^{2} \mathbf{H}, \\
\frac{\partial(\rho \varepsilon)}{\partial t}+\nabla \cdot(\rho \varepsilon \mathbf{v})=-p \nabla \cdot \mathbf{v}+\frac{\mathbf{J}^{2}}{\sigma}, \\
\frac{4 \pi}{c} \mathbf{J}=\nabla \times \mathbf{H} .
\end{gathered}
$$

The variables in the system of equations have the following values: $c$ is the speed of light, $\rho$ is the density of matter, $v$ is the velocity, $H$ is the magnetic field strength, $\varepsilon$ is the internal energy of the mass unit of matter, $\mathbf{J}$ is the electric current density, $\sigma$ is the effective electrical conductivity of the medium. To describe the thermodynamic properties of a substance, the adiabatic equation of state of an ideal gas is used: $p=(\gamma-1) \rho \varepsilon$ where $\gamma$ is the adiabatic exponent. For current calculations, $\gamma=5 / 3$ is chosen, which corresponds to the standard adiabatic exponent for monatomic gases. When writing the system of equations (4.1) - (4.5), Ohm's law was taken into account in the form: $\mathbf{J}=\sigma(\mathbf{E}+\mathbf{v} \times \mathbf{H} / c)$, where $\sigma$ is the electric conductivity. The associated magnetic diffusivity, $\eta_{\mathrm{m}} \equiv c^{2} /(4 \pi \sigma)$, is assumed to be a constant. The gravitational force is zero, $\mathbf{F}^{g}=0$.

The magnetic field can have one of three configurations: the poloidal field $\left(H_{z}, H_{r}\right)$, the toroidal field $H_{\phi}$ or their superposition. In order to fulfill exactly the condition $\nabla \cdot \mathbf{H}=0$, in the calculations instead of the poloidal component of the magnetic field strength $H_{z}$ and $H_{r}$, the toroidal component $A_{\phi}$ vector potential $\mathbf{A}$ is used, $\mathbf{H}=\nabla \times \mathbf{A}$. Early we used this MHD code in the simulations of accretion onto stars with dipole magnetic fields $[12,13]$.

After reducing the system of equations (4.1) - (4.4) to a dimensionless form, a set of characteristic dimensionless parameters appears. First parameter

$$
\beta=\frac{8 \pi P_{0 j e t}}{H_{0}^{2}}=\frac{2}{\gamma} \frac{c_{s 0}^{2}}{V_{A 0}^{2}}
$$

determines the relation between the characteristic values of the pressure of the matter $P_{0 j e t}=$ $\rho_{0 j e t} c_{s 0}^{2} / \gamma$ and the pressure of the magnetic field. Here $\rho_{0 j e t}$ is the density of the inflowing substance at the boundary of the calculated region, $P_{0 j e t}, c_{s 0}$ is the pressure and sound speed in the incoming matter, $H_{0}$ is the magnetic field strength in the region, $V_{A 0}=\frac{H_{0}}{\sqrt{4 \pi \rho_{0 j e t}}}$ is the Alfven velocity of the inflowing substance at the boundary of the calculated region.

The second parameter of the numerical model is the characteristic dimensionless magnetic viscosity

$$
\tilde{\eta}_{M}=\frac{\eta_{M}}{L_{0} V_{A 0}}=\frac{c^{2}}{4 \pi \sigma L_{0} V_{A 0}}=\frac{1}{R e_{M}},
$$

where $\sigma$ is the constant effective conductivity of the plasma; $L_{0}$ is the characteristic size and $V_{A 0}$ is the characteristic Alfven velocity used in determining the Reynolds magnetic number $R e_{M}$. As the characteristic size $L_{0}$ the scale of the problem $R_{\max }$ was taken. As the characteristic velocity $V_{0}$ the Alfven velocity of the inflowing matter is taken at the boundary of the calculated region $V_{A 0}$.

Boundary and initial conditions are described in our recent paper [7] 


\section{Results of numerical simulation}

\subsection{The poloidal magnetic field with $\beta=10^{-3}$}

At this step of the simulation we included an external constant poloidal magnetic field $H$ directed along the normal to the target. We performed a series of calculations for different values of the parameter $\beta$. The characteristic parameter for this case is: $\beta=10^{-3}$ and $\tilde{\eta}_{M}=10^{-5}$. The initial setups for the background density and velocity are the same as those of the simulation described in Section 5.1. The magnetic field is defined as follows: $A_{\phi}=r H, A_{r}=0, A_{z}=0$, $H_{\phi}=0$.
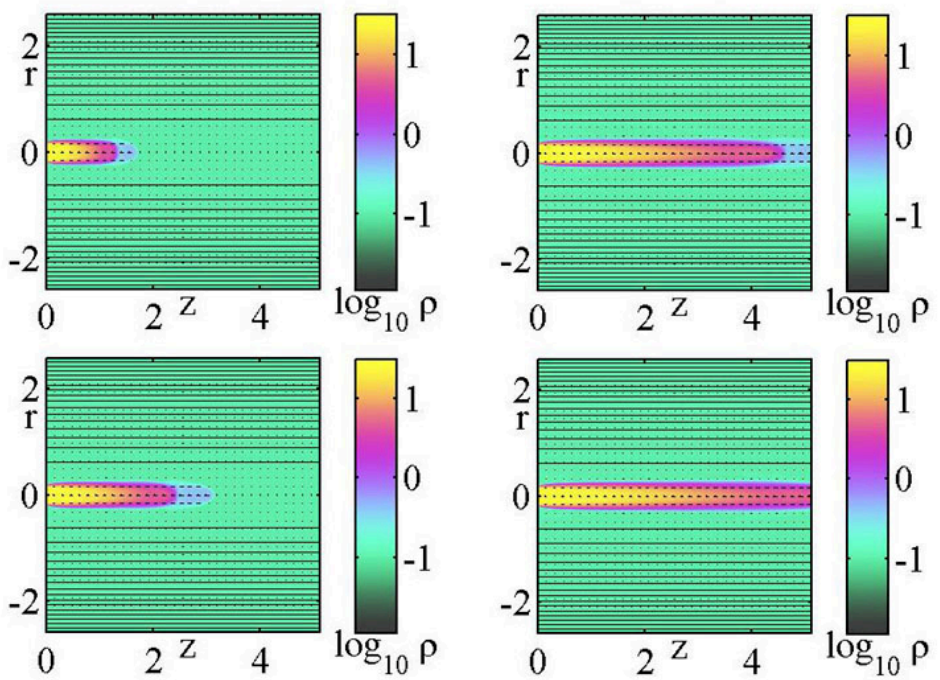

Figure 2: Picture of the matter flow for MHD case $\beta=10^{-3}$ at different moments

The results of simulation for the case $\beta=10^{-3}$ are shown in Figure 2. The color background corresponds to the logarithm of the density. The arrows indicate the velocity vectors. Solid lines represent magnetic field lines. It can be seen that the target substance under the action of the thermal energy of the laser beam begins to fly along the axis $Z$ to the opposite wall of the chamber. A shock wave of elongated shape is formed, which moves from the target to the boundary of the chamber, leaving behind a steady outflow. Due to the strong magnetic field, the flux is well-collimated as the substance does not expand in the direction $R$. The collimation by the external (background) gas thermal pressure in the presence of a strong field does not play a role. Thus, in the presence of a sufficiently strong poloidal magnetic field, a sufficiently sharp continuous spot can be observed on the detector.

\subsection{The poloidal magnetic field with $\beta=10^{-1}$}

In the next simulation, we reduced the magnetic field. The characteristic parameters for this case are: $\beta=10^{-1}$ and $\tilde{\eta}_{M}=0^{-5}$ The initial setups for the background density, velocity, and magnetic fields are the same as those of the simulation described in Section 5.2 except $\beta$. The results of simulation for the case $\beta=10^{-1}$ are shown in Figure 7 . 

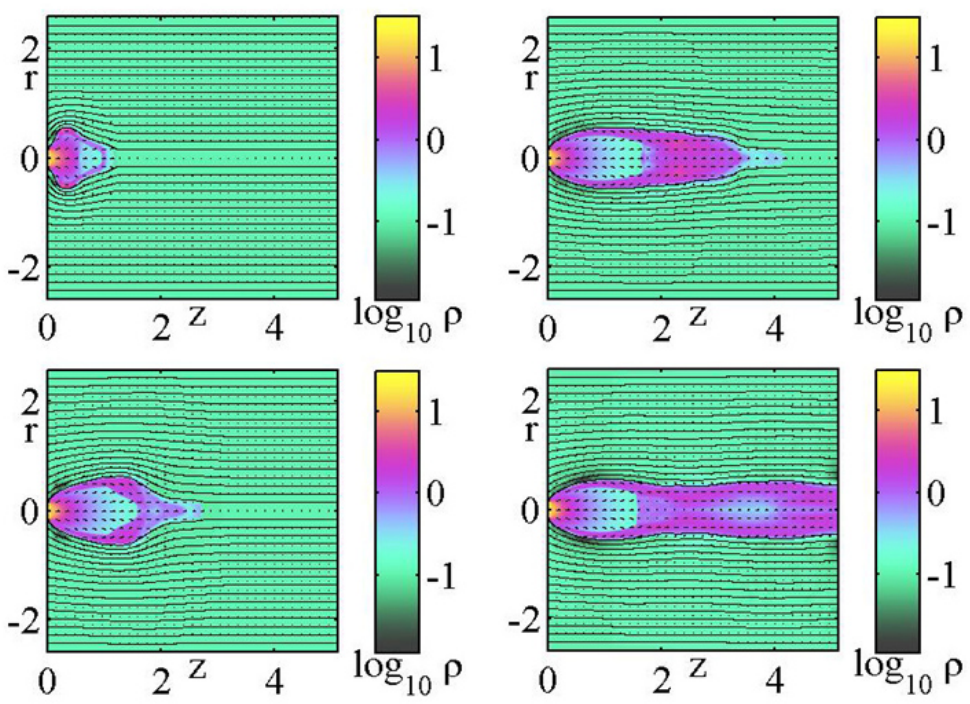

Figure 3: Picture of the matter flow for MHD case $\beta=10^{-1}$ at different moments.

The color background corresponds to the logarithm of the density. The arrows indicate the velocity vectors. Solid lines represent magnetic field lines. It can be seen that after the release of matter it begins to expand in all directions, but then the flow is collimated by a magnetic field. The influence of the external gas thermal pressure on the collimation of the flow in this case is insignificant. Inside the flow, a shock wave is formed at a distance of $z \approx 1.6$.

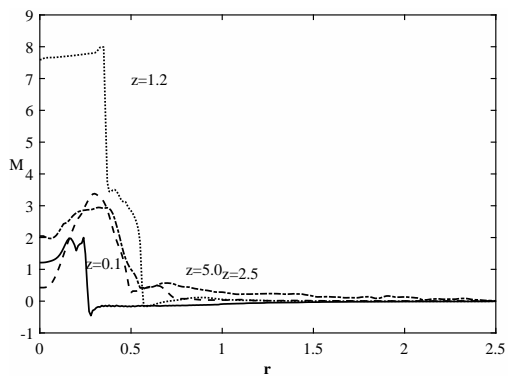

Figure 4: The distribution of the Mach number $M=v / c_{s}$ along $\mathrm{r}$ for different distances from the target $\mathrm{z}, \beta=10^{-1}$.

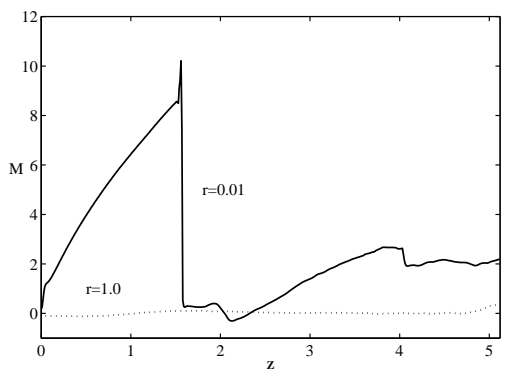

Figure 5: The distribution of the Mach number $M=v / c_{s}$ along $\mathrm{z}$ for different distances from the $r$-axis $\beta=10^{-1}$.

Figure 4 shows the Mach number $M=v / c_{s}$ as a function of the distance from the target. Figure 5 shows the Mach number $M=v / c_{s}$ as a function of the distance from the $r$-axis. Both figures correspond to the moment of the forth epoch in Figure 6. We can see that the velocity of matter rises to a distance of $z \approx 1.6$ and reaches a value of $M \approx 10$. After the passage of the shock wave, the velocity of matter falls sharply to $M \approx 0.5$, and then again begins to increase to $M \approx 2$ at a distance of $z \approx Z_{\max }$.

Figure 6 shows the flux density along $\mathrm{R}$ as a function of the distance from the target at 


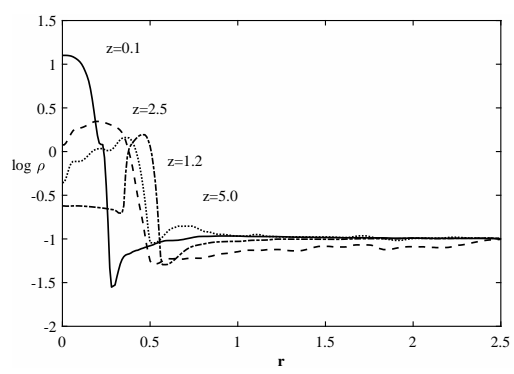

Figure 6: The flux density along $r$ as a function of the distance from the target at different distances $z$ from the target surface for the case $\beta=10^{-1}$.

different distances $\mathrm{z}$ from the target surface for the case $\beta=10^{-1}$. A thin solid line corresponds to the flux density at the boundary of the target. The dot-dashed line corresponds to the distance $z=1.2$, the dashed line corresponds to the density in the middle of the chamber, $z=2.5$, the dotted line corresponds to the density near the camera boundary, $z=5.0$. It can be seen that the density distribution in the plasma flow depends on the distance from the target. If at the beginning of the flow the maximum plasma flux density falls on a narrow range comparable to the target dimensions, then in the shock wave region, $z=1.2$, the density distribution forms a clear ring structure. After the passage of the shock wave, the density distribution becomes more uniform and the ring structure is washed out. From Figures 4 and 6, we can determine the angle of the cone of the flow at the distance from the strongest shock wave. It is approximately equal to $\theta \approx 40$.

We can see from the simulations that near the detector a cavity is formed. It is bounded by a shock wave. The size of the cavity depends on the magnitude of the magnetic field H. Similar results were obtained in work Albertazzi B. et al. [4]. This leads to the formation of ring structures, which are observed in the experiment. In Figure 7 it is shown how the angle of these structures depends on the magnitude of the magnetic field. With a weak field (and also with its absence), this angle is large (left figure). With an increase in the field, the dimension of the cavity and, accordingly, the angle of the ring structure decreases, and then a solid spot is observed instead of the ring (right figure).
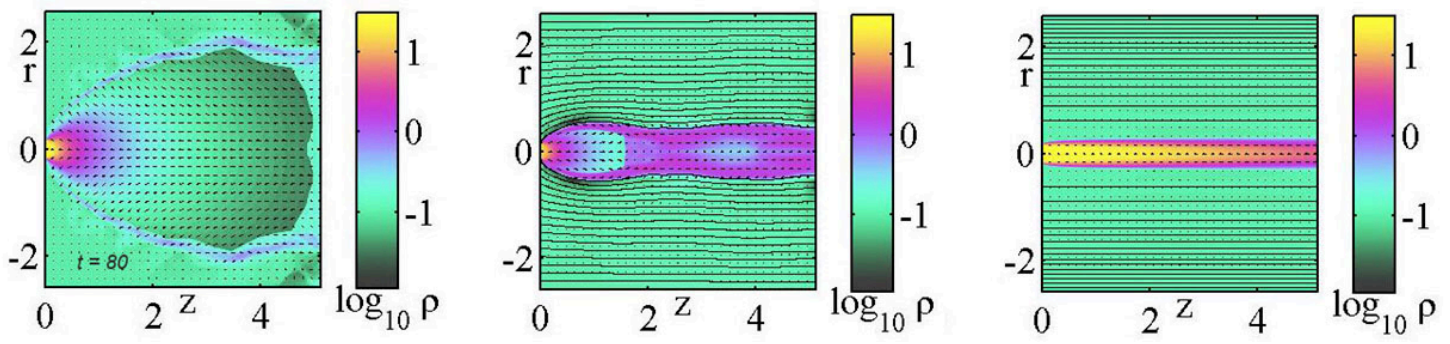

Figure 7: Picture of the matter flow for HD case and MHD cases $\beta=10^{-1}$ and $\beta=10^{-3}$ 


\section{Conclusions}

The paper presents the results of MHD simulation of magnetized supersonic jets. It is shown that in the absence of a magnetic field, a substantial expansion of the jet occurs. In the presence of a strong poloidal magnetic field, the jet expands only slightly, which confirms the possibility of a magnetic collimation mechanism for astrophysical jets. Calculations have shown that under certain values of the poloidal magnetic field ring structure may form. The opening angle of these structures approximates the results of the laboratory experiment $\left(\approx 40^{\circ}\right)$. Thus, a comparison of the results of the laboratory experiment and the numerical calculations of magnetized jets in this paper, as well as the results of the work [4], confirm the possibility of the formation of ring structures whose characteristics depend on the magnitude of the magnetic field. Further theoretical and experimental research is needed in this field. Formation and development of jet in a laboratory laser experiment is a complex physical phenomenon involving a large number of different physical processes. Numerical simulation of such an experiment, allowing to account for all possible physical processes, is a complex problem and is currently difficult to implement. We used the approximation of magnetohydrodynamics, as the first step in the study of this problem.

\section{Acknowledgements}

We are grateful to V.V. Saveliev (IPM RAS) for the original version of the MHD simulation program. This work was partially supported by RFBR grant 17-02-00760, 18-02-00619, 18-2921021 and by Russian Academy of Science program P12.

\section{References}

[1] G.S. Bisnovatyi-Kogan, Stellar Jets and Bipolar Outflows, Dordrecht: Kluwer. Eds. L. Errico, and Alberto A. Vittone. Astrophys. Space Sci. Library, 186, 369, (1993)

[2] W. Fu, E.P. Liang, P. Tzeferacos, D.Q. Lamb, Creation of magnetized jet using a ring of laser beams, High Energy Density Physics, 17, 42, (2015)

[3] K. N. Mitrofanov et.al., Properties of the distribution of azimuthal magnetic field in a plasma flow during laboratory simulations of astrophysical jets in a plasma-focus installation, Astronomy Reports, 61, 138, (2017)

[4] B. Albertazzi et al., Laboratory formation of a scaled protostellar jet by coaligned poloidal magnetic field, Science, 346, 325, (2014)

[5] V. S. Belyaev, V. I. Vinogradov, A. P. Matafonov, Generation of MeV photons and protons in laser picosecond plasmas, Nuclear physics, 71, 466, (2008)

[6] D.D. Ryutov, R.P. Drake, B.A. Remington, Criteria for scaled laboratory simulations of astrophysical MHD phenomena, ApJS, 127, 465, (2000).

[7] V. S. Belyaev et al., Numerical Simulations of Magnetized Astrophysical Jets and Comparison with Laboratory Laser Experiments, Astronomy Reports, 3, (2018)

[8] V.I. Mazhukin, A.V. Shapranov, M.M. Demin, A.A. Samokhin, A.E. Zubko, Molecular dynamics modelling of nanosecond laser ablation: transcritical regime, Mathematica Montisnigri, 38, 3, (2017) 
[9] V.I. Mazhukin, M.M. Demin, A.V. Shapranov, High-speed laser ablation of metal with pico- and subpicosecond pulses, Appl. Surf. Sci., 302, 6, (2014)

[10] L. D. Landau, E. M. Lifshitz, Electrodynamics of Continuous Media (New York: Pergamon), chap. 8, (1960)

[11] V. T. Zhukov, A. V. Zabrodin, O. B. Feodoritova, A method of solving the two-dimensional equations of the dynamics of a heat-conducting gas in regions of complicated shape, Comp. Maths. Math. Phys., 33, No. 8, 1099 (1993)

[12] O.D. Toropina, M.M. Romanova, Yu.M. Toropin, \& R. V. E. Lovelace, Spherical Bondi Accretion onto a Magnetic Dipole, ApJ, 561, 964, (2001)

[13] O.D. Toropina, M.M. Romanova \& R. V. E. Lovelace, Bondi-Hoyle Accretion onto Magnetized Neutron Star, MNRAS, 420, 810, (2012) 